\title{
L-Proline Microinjected into the Rat Ventrolateral Medulla Induces a Depressor Response Distinct from L-Glutamate
}

\author{
Y. TAKEMOTO \\ Department of Neurophysiology, Division of Integrated Medical Science, Programs for Biomedical Research, \\ Graduate School of Biomedical Sciences, Hiroshima University, Hiroshima, 734-8551 Japan
}

\begin{abstract}
The neurotransmitter candidate Lproline elicits changes in the cardiovascular system via actions in the brainstem. However, its action have not yet been determined in the ventrolateral medulla (VLM), a brain region critical in mediating vasomotor sympathetic nervous system responses. Microinjections of L-glutamate produce depressor responses in the caudal (C) VLM, but pressor responses in the rostral (R) VLM and the caudal pressor area (CPA) in the far caudal CVLM. The present study tested whether microinjections of L-proline in the VLM produce a pattern of hemodynamic responses distinct from that of L-glutamate. Urethaneanesthetized rats received arterial catheters and were implanted with flow probes around the abdominal aorta (supplies hindquarters). The surface of each rat's VLM was then exposed. LProline induced dose-dependent depressor responses in the CVLM $(0.003-1.0 \mathrm{M}, 34 \mathrm{nl})$,
\end{abstract}

but did not induce hemodynamic responses in sites of the RVLM $(0.01-1.0 \mathrm{M}, 34 \mathrm{nl})$ that responded to L-glutamate $(0.01 \mathrm{M}, 34 \mathrm{nl})$. LProline injections $(0.1 \mathrm{M}, 34 \mathrm{nl})$ induced rapid and consistent depressor responses correlated with coincident decreases in hindquarter resistance (arterial blood pressure/flow) in the CVLM and CPA, but only inconsistent responses in a few sites in the RVLM. In summary, L-proline induced a distinct pattern of depressor responses preferentially in caudal regions of the VLM, and these depressor effects were associated with decreases in hindquarter resistance. These findings indicate that L-proline may have unique roles including cardiovascular regulation independently from L-glutamate, especially in caudal region of the VLM, via a mechanism that involves altering hindquarter resistance. [The Japanese Journal of Physiology 54: 339-345, 2004]

Key words: L-proline, ventrolateral medulla, arterial blood pressure.

The The nonessential amino acid L-proline has been reported as a neurotransmitter candidate in the central nervous system $[1,2]$. In the rodent brain, it is synthesized enzymatically from pyrroline 5-carboxylic acid by pyrroline 5-carboxylate reductase (EC1.5.1.2) [3]. A potassium stimulation of neurons in the brain slices [4] and of cortical synaptosomes [5] can induce the release of radiolabeled L-proline. An expression of a proline transporter cloned by cDNA was reported in the rat brain [6]. L-Proline exerts various electrophysiological actions in the spinal cord $[1,7,8]$, the cerebellum [9], and the cerebral cortex [1].

The brain application of L-proline alters arterial blood pressure (ABP) [10-13]. Namely, an intra-cisternal injection of L-proline in the freely moving rat increases ABP [10]. This effect is mediated through vasopressin release [11] and via central ionotropic excitatory amino acid receptors [12]. The microinjection of L-proline into the nucleus tractus solitarii of the anesthetized rat induces depressor and bradycardiac actions similar to those seen with L-glutamate

Received on January 14, 2004; accepted on June 29, 2004

Correspondence should be addressed to: Yumi Takemoto, Kasumi 1-2-3, Minami-ku, Hiroshima, 734-8551 Japan. Tel/Fax: +81-82-2575128, E-mail: yumitake@hiroshima-u.ac.jp 
[13]. However, the responses to both amino acids have been differentiated by kynurenate, an ionotropic excitatory amino acid receptor antagonist. Actions by Lproline were blocked, but those by L-glutamate augmented [13]. This finding suggests that L-proline may regulate cardiovascular function by a mechanism different from that of L-glutamate. The ventrolateral medulla (VLM) is known to be important in the control and integration of neural output of the vasomotor system [14-16]. An injection of L-glutamate produces a pressor response in the rostral (R) VLM and a depressor response in the caudal (C) VLM [14-16]. The RVLM is thought to determine the basal sympathetic vascular tone and to be inhibited directly by the CVLM [14-16]. Central GABAergic receptor stimulation has been reported to significantly and sympathetically affect the resting vascular tone in the abdominal aorta (that supplies blood to the hindquarters) [17], suggesting that the hindquarter vascular resistance is important in maintaining and controlling ABP. Therefore the present study evaluated the responses of $\mathrm{ABP}$ and hindquarter resistance with a microinjection of L-proline into the VLM of anesthetized rats, relative to responses observed with Lglutamate injection, to test whether microinjections of L-proline in the VLM produce a pattern of hemodynamic response distinct from that of L-glutamate.

\section{METHODS}

All protocols and surgical procedures used in this study were performed in accordance with the Guiding Principles for the Care and Use of Animals approved by the Council of the Physiological Society of Japan and with Guideline for Animal Experiment in Hiroshima University and the Committee of Research Facilities for Laboratory Animal Science, Natural Science Center for Basic Research and Development (N-BARD), Hiroshima University.

Operation and preparation of animals. The present study employed a ventral approach to the medulla because preliminary studies revealed less variability with a ventral than with a dorsal approach. Experiments were conducted on 46 male Wistar rats, weighing 330-370 g. The animals were anesthetized with urethane (1.0-1.3 g/kg, I.P.), and supplemental doses $(75 \mathrm{mg} / \mathrm{kg} /$ injection, I.V.) were given intermittently to maintain the anesthesia. An electromagnetic flow probe of $2 \mathrm{~mm}$ in inner diameter (Nihon Kohden, type FC) was implanted around the terminal aorta just rostral to the bifurcation of the common iliac arteries. The animals were placed in a supine position on a stereotaxic frame (Narishige) with the nose bar set $4 \mathrm{~mm}$ below the level of the interaural line. A tracheotomy and an insertion of a tracheal cannula coated with atropine sulfate ointment (Santen) were performed. To leave the abdominal aortic flow intact for measurement, the carotid artery was catheterized with polyethylene tubing (PE50), and $\mathrm{ABP}$ was monitored via a pressure transducer. The rat has a bypassing route of the flow from the basilar artery to the carotid artery [18]; thus the lack of flow from the carotid artery after its occlusion is compensated for by flow from the basilar artery. ABP, heart rate (HR) derived from blood pressure waves, and terminal aortic flow were recorded on a pen-writing oscillograph (Nihon Kohden). The rats were ventilated by a rodent respirator (Shinano) to maintain normocapnia $\left(\mathrm{PaCO}_{2}: 35-45 \mathrm{mmHg}\right)$. End-expiratory $\mathrm{CO}_{2}$ was monitored (Capnocheck plus), and these values were calibrated by i-STAT (Fuso) with blood gas measurement. A microscope was used to observe the brain stem region. The ventral surface of the medulla oblongata was exposed by opening a window in the basioccipital bone. The dura and arachnoid were opened carefully to avoid tearing surface vessels. The rats were immobilized by an I.V. infusion of pancuronium bromide (Sankyo) at a rate of $0.4 \mathrm{mg} / \mathrm{kg} / \mathrm{h}$. After the induction of neuromuscular blockade, an adequate depth of anesthesia was assessed according to ABP stability and/or the absence of a withdrawal response to a firm toe pinch during a stoppage of the infusion of neuromuscular blockade. The rectal temperature of the rats was maintained at $37 \pm 0.5^{\circ} \mathrm{C}$ by a heating mat or a cooling bag.

Preparation for microinjections. For microinjections of solutions into the ventral surface of the medulla, a glass micropipette (20-30 $\mu$ m o.d. at the tip) was prepared and mounted onto a pipette holder. The other end of the micropipette also had a narrowed tip connected tightly to polyethylene tubing and a Hamilton microsyringe attached to a micromanipulator. The graduation of the micromanipulator had been calibrated previously by the microsyringe. The micropipette attached tubing and syringe were filled with distilled water. Amino acids were dissolved in artificial cerebrospinal fluid [13]. Injections of several doses of amino acid containing solutions, L-glutamate or L-proline, were delivered through the same micropipette into test sites to investigate the dose-response functions of each acid. The interval between injections was longer than $5 \mathrm{~min}$. Injections were performed $0.5-1.0 \mathrm{~mm}$ in depth from the surface of the VLM where there were no arteries.

Experiments. Protocol 1: Functional determination of RVLM and CVLM by L-glutamate. The 
depressor area in the CVLM (0.7-1.0 $\mathrm{mm}$ in depth) and pressor area in the RVLM (0.5-0.7 mm deep) on the ventral surface were functionally defined by 112 trial injections of L-glutamate $(0.01 \mathrm{M}, 34 \mathrm{nl})$ in 12 rats. The caudolateral edge of the beginning of the basilar artery (Fig. 1) was set as the zero point [19]. The distance of the rostral end of the second rootlet of cranial nerve XII was $1.11 \pm 0.04 \mathrm{~mm}$ rostral to the zero point (mean $\pm \mathrm{SE}$, ranging from 0.6 to 1.55 $\mathrm{mm}, 46$ rats).

Protocol 2: Dose-response relationship of L-proline. The dose-response relationship of L-proline was investigated in the VLM. L-Proline was injected into the CVLM (0.003 M to $1.0 \mathrm{M}, 34 \mathrm{nl}, 5$ rats), and into the RVLM $(0.01 \mathrm{M}$ to $1.0 \mathrm{M}, 8$ rats $)$ where pressor responses were observed after L-glutamate injection.

Protocol 3: Mapping of cardiovascular responses to L-proline. The most effective dose of L-proline $(0.1$ M, $34 \mathrm{nl}$ ) was injected into sites throughout the VLM of 16 rats (219 trials) to map cardiovascular responsive sites. The responses of $\mathrm{ABP}, \mathrm{HR}$, and hindquarter flow to microinjections of L-proline were monitored. Hindquarter resistance data (HQR) were obtained by dividing ABP by hindquarter flow.

After completion of the experiments, the animals were killed with an overdose of sodium pentobarbital (100 mg/kg I.V.; Dainippon).

\section{RESULTS}

Table 1 summarizes the changes in the cardiovascular variables obtained in the L-glutamate and L-proline mapping experiments.
Determination of RVLM, CVLM, and CPA by L-glutamate. A map of loci that mediated pressor and depressor responses to L-glutamate injections was developed (Fig. 1). The sites that produced pressor responses $(>10 \mathrm{mmHg})$ were situated 1.5 to 4.0 $\mathrm{mm}$ rostral to and 1.0 to $2.8 \mathrm{~mm}$ lateral to the zero point; this area was identified as the RVLM. The sites that produced depressor responses $(<-10 \mathrm{mmHg})$ were situated 0.5 to $1.5 \mathrm{~mm}$ rostral to and 1.5 to 2.3 $\mathrm{mm}$ lateral to the zero point; this area was identified as the CVLM. Additionally, several sites caudal to the CVLM ( -0.5 to 0.5$)$ induced a pressor response ( $>10 \mathrm{mmHg}$ ). These sites seem to belong to the caudal pressor area (CPA) described by Gordon and McCann [20].

Dose-response relationship of L-proline in the CVLM and RVLM. L-Proline injections (0.003 to $0.1 \mathrm{M}, 34 \mathrm{nl}$ ) into the CVLM induced a dosedependent depressor response, but doses higher than $0.1 \mathrm{M}$ L-proline showed a plateau response (Fig. 2). In contrast, L-proline (0.01-1.0 M) did not affect ABP in RVLM sites responsive to $0.01 \mathrm{M}$ L-glutamate injection (Fig. 3).

Mapping of ABP response to L-proline. The loci of pressor and depressor responses to L-proline (0.1 M, $34 \mathrm{nl})$ over the VLM were mapped (Fig. 4). In the VLM, L-proline injection elicited a depressor response $(<-10 \mathrm{mmHg})$ at 57 sites, a pressor response ( $>10 \mathrm{mmHg}$ ) at 15 sites, and no response at 147 sites. The sites showing a depressor response to L-proline were situated -0.7 to $2 \mathrm{~mm}$ rostral to and 1.3 to 2.4 $\mathrm{mm}$ lateral to the zero point, exceptionally contain-

Table 1. Summary of cardiovascular variables during the mapping ventrolateral medulla mediated changes.

\begin{tabular}{|c|c|c|c|c|}
\hline & $\begin{array}{l}\operatorname{ABP}(n) \\
\mathrm{mmHg}\end{array}$ & $\begin{array}{c}\mathrm{HR}(n) \\
\text { beats/min }\end{array}$ & $\begin{array}{c}\operatorname{HQR}(n) \\
\mathrm{mmHg} \min 100 \mathrm{~g} / \mathrm{ml}\end{array}$ & $\begin{array}{c}\operatorname{HQF}(n) \\
\mathrm{ml} / \mathrm{min} / 100 \mathrm{~g}\end{array}$ \\
\hline \multicolumn{5}{|l|}{ L-glutamate } \\
\hline RVLM & $21 \pm 2.2(36)$ & $9.3 \pm 1.4(34)$ & $11 \pm 3.0(34)$ & $0.01 \pm 0.03(34)$ \\
\hline CVLM & $-20 \pm 1.9(14)$ & $-6.2 \pm 1.1(14)$ & $-7.2 \pm 2.7(13)$ & $-0.14 \pm 0.08(13)$ \\
\hline CPA & $19 \pm 3.3(4)$ & $15 \pm 11(4)$ & $8.6 \pm 7.0(4)$ & $-0.03 \pm 0.06$ \\
\hline \multicolumn{5}{|l|}{ L-proline } \\
\hline RVLM & $15 \pm 1.2(13)$ & $3.5 \pm 1.2(13)$ & $7.4 \pm 1.0(11)$ & $0.08 \pm 0.03(11)$ \\
\hline & $-15 \pm 1.7(4)$ & $-1.8 \pm 0.63(4)$ & $-11 \pm 3.1$ (3) & $-0.18 \pm 0.18$ \\
\hline \multirow{2}{*}{ CVLM } & $15(1)$ & $3(1)$ & $3.6(1)$ & $-0.11(1)$ \\
\hline & $-18 \pm 1.2(34)$ & $-5.0 \pm 0.63(34)$ & $-9.8 \pm 1.3(31)$ & $0.05 \pm 0.08$ \\
\hline \multirow{2}{*}{ CPA } & $30(1)$ & $12(1)$ & $39(1)$ & $-0.32(1)$ \\
\hline & $-19 \pm 1.5(19)$ & $-5.9 \pm 0.93(19)$ & $-13 \pm 2.0(18)$ & $-0.27 \pm 0.29(18)$ \\
\hline
\end{tabular}

Mean \pm standard error. RVLM: rostral pressor activated area; CVLM: caudal depressor area; and CPA: caudal pressor

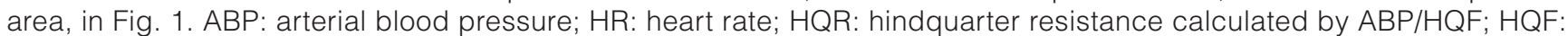
hindquarter flow; and $n$ : the number of trials. A different number in $n$ means that cases failed to obtain the values in determination of $\mathrm{HR}$ and $\mathrm{HQF} / \mathrm{HQR}$. Basal values were $94 \pm 1.0 \mathrm{mmHg}(n=126$ : total number in the table) in ABP, 480 \pm 2 beats/min (124) in HR, $55 \pm 1.7 \mathrm{mmHg} \min 100 \mathrm{~g} / \mathrm{ml}(116) \mathrm{in} \mathrm{HQR}$, and $1.83 \pm 0.07 \mathrm{ml} / \mathrm{min} / 100 \mathrm{~g}(116)$ in HQF. Mean and standard errors of changes in HR was $-5.0 \pm 0.5$ beats/min (57) when L-proline produced the depressor change. 


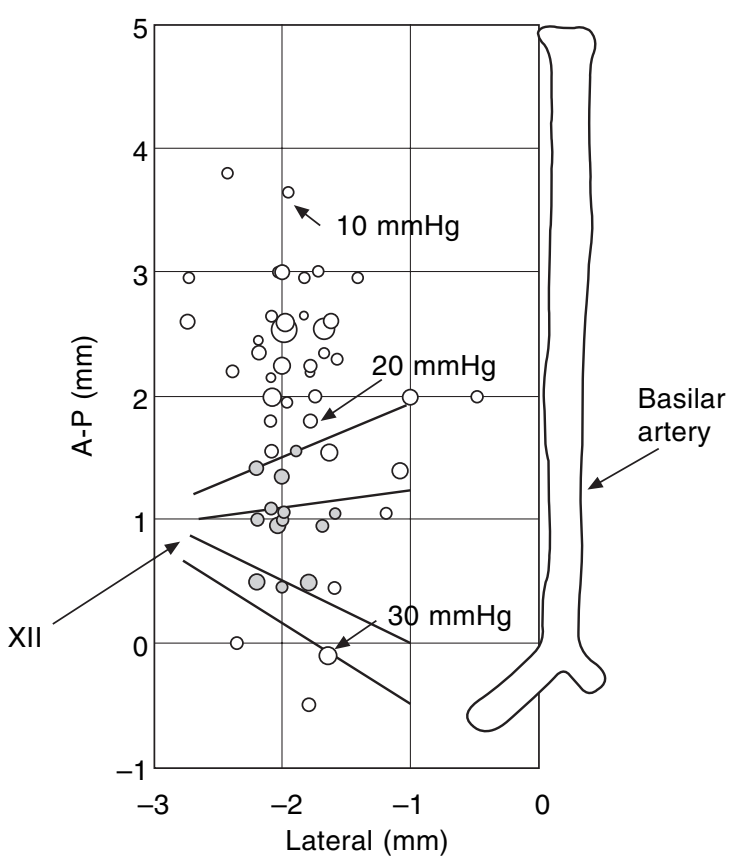

Fig. 1. Mapping of sites showing arterial blood pressure $(A B P)$ responses to L-glutamate $(0.01 \mathrm{M}, 34 \mathrm{nl})$ injection into the ventral medulla of 12 rats. Open circles indicate sites for an increase in ABP greater than 10 $\mathrm{mmHg}$. Closed circles indicate sites for a decrease in ABP greater than $10 \mathrm{mmHg}$. The circle sizes are proportional to degrees of response. RVLM: rostral pressor-activated area; CVLM: caudal depressor area; and CPA: caudal pressor area. A-P means anterior-posterior coordination from the beginning of the basilar artery direction. XII: The XII cranial nerves.

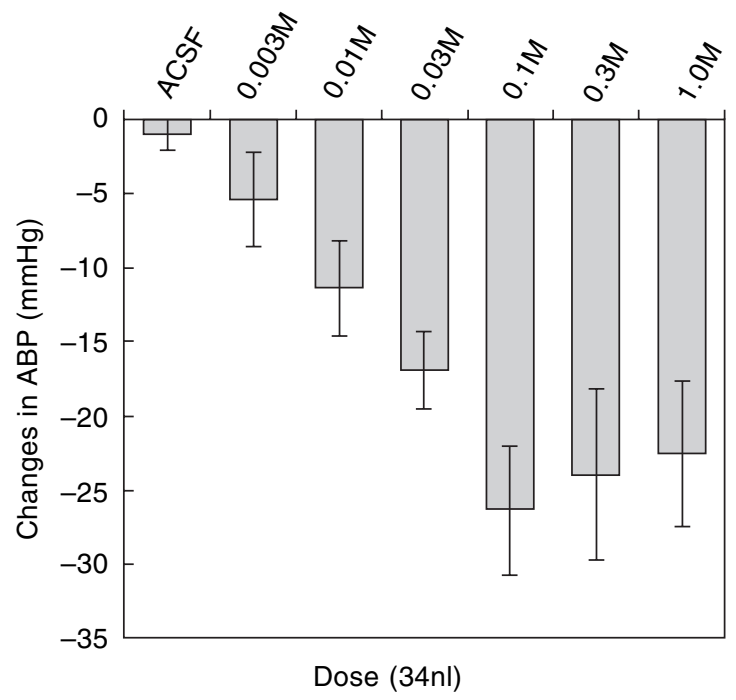

Fig. 2. The dose-response relationship of L-proline injected into the caudal area of the ventrolateral medulla of $\mathbf{5}$ rats. ABP: arterial blood pressure. Bars indicate standard errors. ACSF: artificial cerebral spinal fluid.

ing 2 pressor response sites. The sites showing a pressor response were situated $2-4 \mathrm{~mm}$ rostral to and $1.7-2.4 \mathrm{~mm}$ lateral to the zero point. In the

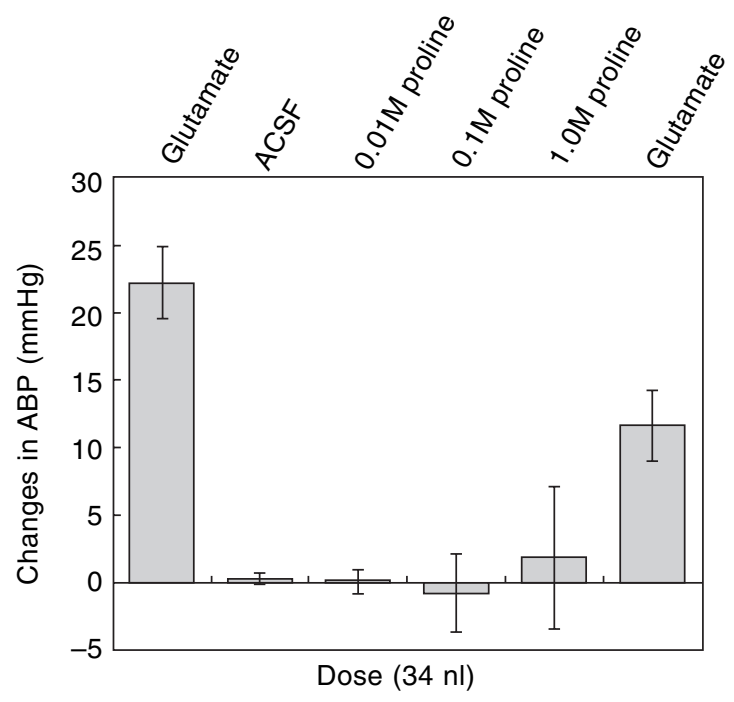

Fig. 3. Responses to L-proline and L-glutamate injected into the RVLM of 8 rats. ABP: arterial blood pressure. Glutamate was injected before (left) and after (right) proline injections. Bars indicate standard error. ACSF: artificial cerebrospinal fluid.

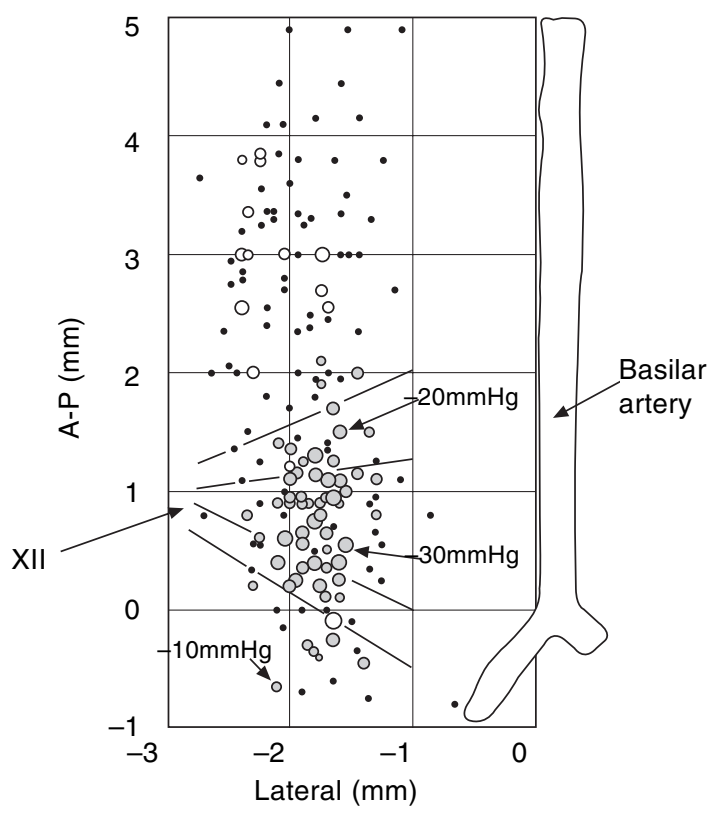

Fig. 4. Mapping of sites showing arterial blood pressure $(A B P)$ responses to L-proline $(0.1 \mathrm{M}, 34 \mathrm{nl})$ injection into the ventral medulla of 16 rats. Open circles indicate sites for an increase in $\mathrm{ABP}$ greater than $10 \mathrm{mmHg}$. Closed circles indicate sites for a decrease in ABP greater than $10 \mathrm{mmHg}$. The circle sizes are proportional to degrees of response. Dots indicate sites for no change or changes less than $10 \mathrm{mmHg}$ in ABP. A-P means anterior-posterior co-ordination from the beginning of the basilar artery direction. XII: The XII cranial nerves.

RVLM defined by the response to L-glutamate (Fig. 1), 13 sites had a pressor response and 4 sites a depressor response. In the CVLM, 34 sites had a 


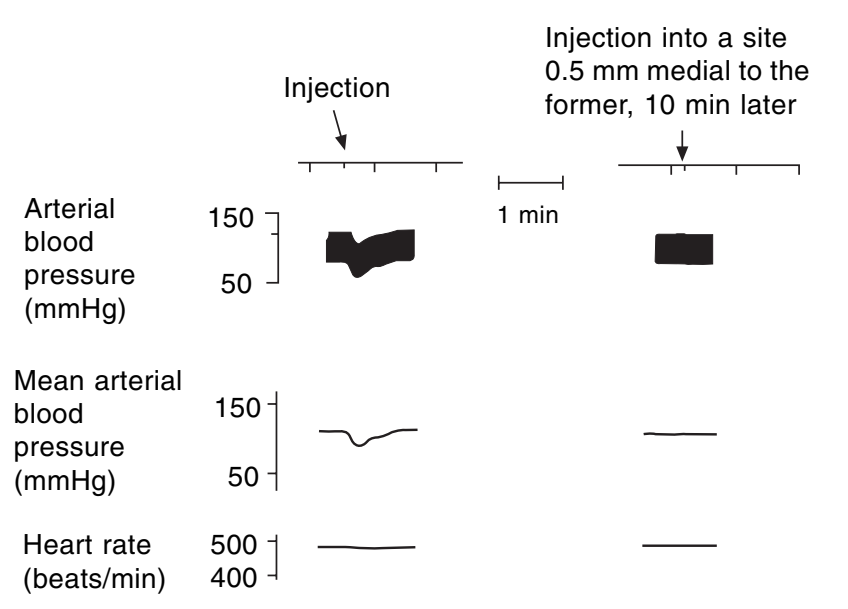

$\left.\begin{array}{ll}\text { Hindquarter } & 3 \\ \text { blood flow } & 0\end{array}\right]=$

Fig. 5. Traces of cardiovascular responses to $0.1 \mathrm{M}$ proline (34 $\mathrm{nl})$ injected into a site in the caudal part of the ventrolateral medulla and a site $0.5 \mathrm{~mm}$ medial to the former.

depressor response and 1 site a pressor response to L-proline injection. And in the CPA, 19 sites had a depressor response and 1 site a pressor response to L-proline injection. Figure 5 illustrates cardiovascular response traces when $0.1 \mathrm{M} \mathrm{L}$-proline was injected into a depressor site of the CVLM and into an unresponsive site $0.5 \mathrm{~mm}$ medial to the former site. The onset of the depressor action was just after the intraCVLM L-proline injection, and the decreased ABP recovered to the pre injection level within one minute. The pressor response to L-proline injection in the rostral parts of the VLM showed the same quick onset and recovery to the pre-injection level of ABP. An injection of L-proline into the caudal part of the VLM consistently induced a depressor response in every rat tested. However, an injection into the rostral part induced a pressor response inconsistently: some rats showed the pressor response, but others showed no response.

Relationship between changes in ABP and hindquarters resistance. Changes in $\mathrm{ABP}$ can be induced by changes in cardiac output and/or total peripheral resistance and may reflect associated changes in regional vascular resistance. Therefore the contribution of changes in hindquarter resistance to L-proline-induced depressor responses was evaluated. Because there were various levels of changes in $\mathrm{ABP}$ and hindquarter resistance (Table 1), the correlation between these variables was tested. These data revealed a robust correlation $(r=0.772, n=52)$ (Fig.

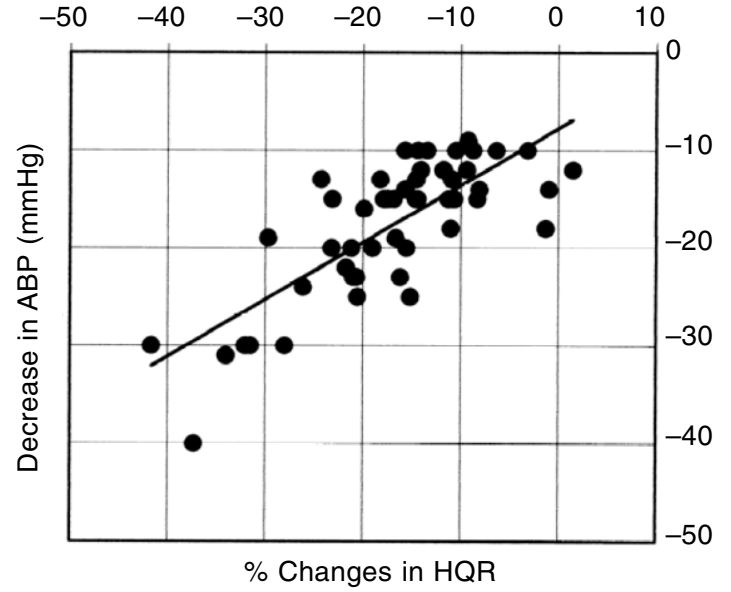

Fig. 6. Relationship between arterial blood pressure (ABP) and change in hindquarter resistance (HQR). Correlation coefficient $=0.772$.

6). The mean change in HR was $-1.0 \%$ (calculated by $-5.0 / 480$ in Table 1 ), suggesting a minor influence of cardiac output on changes in ABP.

\section{DISCUSSION}

The present study demonstrated a depressor action of L-proline preferentially in the CVLM and CPA, but not in the RVLM, of anesthetized rats. Microinjections of L-proline into the CVLM evoked a consistent and dose-dependent depressor response. However, L-glutamate responsive RVLM areas were unresponsive to several doses of L-proline. The values in the mapping of L-proline microinjection revealed a significant correlation between decreases in $\mathrm{ABP}$ and hindquarter resistance. This finding suggests that there is a contribution of sympathetically reduced hindquarter resistance to the decrease in total peripheral resistance and $\mathrm{ABP}$ induced by L-proline stimulation in the CVLM and CPA. The observations of quick ABP decrease onset and recovery after Lproline injection are consistent with the involvement of changes in the sympathetic nervous system [21].

The broad spectrum ionotropic excitatory amino acid receptor antagonist kynurenate has previously been shown to differentiate L-glutamate- and L-proline-induced pressor responses in the cisterna magna [12] and depressor responses in the NTS [13]. Namely, the injection of kynurenate blocked only responses to L-proline, but not to L-glutamate, in both cases. The present study investigated the differing cardiovascular responses to L-proline and Lglutamate. L-Glutamate induced a pressor response both in the RVLM and CPA, but L-proline did not produce a consistent pressor response in the RVLM and evoked a depressor response in the CPA. These 
findings indicate that the responses to L-proline are not mediated by L-glutamate enzymatically converted from L-proline. Instead, L-proline has a distinct response pattern in the VLM. L-Glutamate affects some 20 receptors for excitatory amino acid ligands [22]. L-Proline may bind a subset of excitatory amino acid receptors with a selective distribution over the medulla. Another possibility is that L-proline may act on a distinct L-proline receptor population.

The stimulation of L-proline in the cisterna magna of the conscious rat produces a pressor action mainly via vasopressin release [11]. The present study did not show a consistent glutamate like pressor response to L-proline injection into the VLM, but it did reveal a variable pressor response in the RVLM. The reason for the inconsistent response is not known. Furthermore, the CVLM that includes sites such as $\mathrm{A} 1$ and $\mathrm{C} 1$ where vasopressin can be released after a stimulation induced depressor response instead of a pressor response. Anesthesia could contribute to this difference in responses between conscious and anesthetized conditions. General anesthetics augment the $\mathrm{GABA}_{\mathrm{A}}$ receptor function by prolonging the opening time of chloride channels [23] and thus would enhance afferent GABAergic inhibition of the hypothalamus. Therefore L-proline-induced excitation may be insufficient to cause vasopressin release under anesthesia. Alternatively, this discrepancy may be due to more complicated vasopressin release regulating neural circuits and/or other L-proline responsive sites activated in the conscious state.

The depressor eliciting concentration of L-proline $(0.003$ to $0.1 \mathrm{M})$ in the CVLM was smaller than that in the NTS $(0.05-0.4 \mathrm{M})$ [13], probably indicating a more important role for L-proline in the CVLM than in the NTS. In the circuit of the baroreceptor reflex, the CVLM is thought to send inhibitory projections to the RVLM, a key area in the regulation of the vasomotor sympathetic activity in the CNS [14-16]. It was recently reported that the CVLM contains two functionally distinct regions, one related to the baroreceptor reflex and the other to a non baroreceptor reflex [24], and that neurons projecting to the spinal cord, hypothalamus, and other regions are intermingled in these parts in the CVLM [25]. Furthermore, the RVLM appears to be regulated by inhibitory and excitatory projections from the CVLM [26]. Other studies suggest CVLM involvement in depressor responses to the activation of bed nucleus of the stria terminalis [27] and the central nucleus of amygdala [28]. There is not yet a comprehensive understanding of the role of CVLM neurons in cardiovascular system regulation. The present findings suggest that the neurotransmitter candidate L-proline may have a unique role in the CVLM. Further studies on the preferential and limited properties of L-proline responsive loci in the CVLM in anesthetized rats should elucidate the role of the CVLM.

In conclusion, the present study showed that L-proline induces a depressor response preferentially in caudal parts of the VLM in anesthetized rats, in part through a decrease in hindquarter resistance. Responses to L-proline across the VLM differed substantially from those to L-glutamate. Thus the present findings suggest that the neurotransmitter candidate L-proline has dissociable and functionally distinct roles in the central nervous system including the CVLM.

The author sincerely thanks Dr. Hitoshi Kawano, Department of Anatomy and Physiology, Saga University, for his critical and constructive suggestions on the manuscript, and Professor Nobukuni Ogata, head of our department, for his encouragement to carry out the present project. This work was supported in part by Grantsin-Aid for Scientific Research from the Ministry of Education, Culture, Sport, Science and Technology of Japan and by the Tsuchiya Foundation.

\section{REFERENCES}

1. Felix D, and Kuenzle $H$ : The role of proline in nervous transmission. Adv Biochem Psychopharmacol 15: 165-173, 1976

2. Giacobini E: Imino acids of the brain. In: Handbook of Neurochemistry, 2nd ed., Metabolism in the Nervous System, vol. 3, ed. Lajtha A, Plenum, New York, pp. 583-605, 1983

3. Yoneda $\mathrm{H}$, and Roberts E: A new synaptosomal biosynthetic pathway of proline from ornithine and its negative feedback inhibition by proline. Brain Res 239: 479-488, 1982

4. Mulder AH, and Snyder SH: Potassium-induced release of amino acids from cerebral cortex and spinal cord slices of the rat. Brain Res 76: 297-308, 1974

5. Nickolson VJ: "On" and "Off" responses of $\mathrm{K}^{+}$induced synaptosomal L-proline release: involvement of the sodium pump. J Neurochem 38: 289-292, 1982

6. Fremeau RT Jr, Caron MG, and Blakely RD: Molecular cloning and expression of a high affinity L-proline transporter expressed in putative glutamatergic pathways of rat brain. Neuron 8: 915-926, 1992

7. Ault B, Wang CM, and Yawn BC: L-Proline depolarizes rat spinal motoneurons by an excitatory amino acid antagonist-sensitive mechanism. Br J Pharmacol 92: 319-326, 1987

8. Henzi V, Reichling DB, Helm SW, and MacDermott AB: L-Proline activates glutamate and glycine receptors in cultured rat dorsal horn neurons. Mol Pharmacol 41: 793-801, 1992

9. Zarzecki P, Blum PS, Cordingley GE, and Somjen GG: 
Microiontophoretic studies of the effects of L-proline on neurons in the mammalian central nervous system. Brain Res 89: 187-191, 1975

10. Takemoto Y: Amino acids with central pressor effect in conscious rats. Jpn J Physiol 40: 561-565, 1990

11. Takemoto Y: Regional hemodynamic changes and vasopressin release induced by intracisternal injection of L-proline in the conscious rat. Jpn J Physiol 45: 743-758, 1995

12. Takemoto $Y$ : Kynurenic acid inhibits circulatory responses to intracisternally injected L-proline in conscious rats. Neurosci Let 261: 121-123, 1999

13. Takemoto $Y$ : Depressor and bradycardic actions of Lproline injected into the nucleus tractus solitarii of anesthetized rats. Jpn J Physiol 51: 687-692, 2001

14. Dampney RAL: Functional organization of central pathways regulating the cardiovascular system. Physiol Rev 74: 323-364, 1994

15. Schreihofer AM, and Guyenet PG: The baroreflex and beyond: control of sympathetic vasomotor tone by GABAergic neurons in the ventrolateral medulla. Clin Exp Pharmacol Physiol 29: 514-521, 2002

16. Sved AF, Ito S, Madden CJ, Stocker SD, and Yajima Y: Excitatory inputs to the RVLM in the context of the baroreceptor reflex. Ann NY Acad Sci 940: 247-258, 2001

17. Takemoto Y: Possible contribution of central $\gamma$-aminobutyric acid receptors to resting vascular tone in freely moving rats. Exp Physiol 85: 479-485, 2000

18. Davson H, Welch K, and Segal MB: The physiology and pathophysiology of the cerebrospinal fluid. Churchill Livingstone, Edinburgh and New York, 1987

19. Kuwaki T, Cao WH, Unekawa M, Terui N, and Kumada $M$ : Endothelin-sensitive areas in the ventral surface of the rat medulla. J Auton Nerv Syst 36: 149-158, 1991
20. Gordon FJ, and McCann LA: Pressor responses evoked by microinjections of L-glutamate into the caudal ventrolateral medulla of the rat. Brain Res 457: 251-258, 1988

21. Takemoto Y: Regional hemodynamic responses to exogenous catecholamines and vasoactive peptides in conscious rats. Jpn J Physiol 49: 185-191, 1999

22. Ozawa S, Kamiya H, and Tsuzuki K: Glutamate receptors in the mammalian central nervous system. Prog Neurobiol 54: 581-618, 1998

23. Franks NP, and Lieb WR: Molecular and cellular mechanisms of general anesthesia. Nature 367: 607-614, 1994

24. Cravo SL, Morrison SF, and Reis DJ: Differentiation of two cardiovascular regions within caudal ventrolateral medulla. Am J Physiol 261: R985-994, 1991

25. Verberne AJM, Stornetta RL, and Guyenet PG: Properties of $\mathrm{C} 1$ and other ventrolateral medullary neurones with hypothalamic projections in the rat. $J$ Physiol 517.2: 477-494, 1999

26. Natarajan M, and Morrison SF: Sympathoexcitatory CVLM neurons mediate responses to caudal pressor area stimulation. Am J Physiol 279: R364-374, 2000

27. Giancola SB, Roder S, and Ciriello J: Contribution of caudal ventrolateral medulla to the cardiovascular responses elicited by activation of bed nucleus of the stria terminalis. Brain Res 606: 162-166, 1993

28. Salome N, Viltart $O$, Leman $S$, and Sequeira $H$ : Activation of ventrolateral medullary neurons projecting to spinal autonomic area after chemical stimulation of the central nucleus of amygdala: a neuroanatomical study in the rat. Brain Res 890: 287-295, 2001 
